\title{
Durable miniaturized bioelectronics
}

\author{
Advances in materials science and engineering enable ever-smaller and more reliable \\ bioelectronic devices.
}

At the end of his famous speech from 1959, 'There's Plenty of Room at the Bottom', Richard Feynman envisaged that patients would eventually be able to "swallow the surgeon whole", meaning that small medical robots would be able to travel inside the human body to heal tissues and organs. The first implantation of a cardiac pacemaker in a patient, reported the same year, seemed to be the start of Feynman's vision being realized. Since then, miniaturization has led to a progressive increase in the number of components in cardiac pacemakers, enabling them to deliver a regular pulse and to alter it according to the patient's activity level. Miniaturization, which in part has been enabled by the self-fulfilling validation of Moore's Law - the projection, initially made in 1965 and now trundling to an end (go.nature.com/2mrZvi2), that the number of transistors in an integrated circuit would continue to double approximately every two years - is in fact the overarching theme that has motivated many advances in the design and fabrication of electronic devices for restoring function to damaged organs or tissues.

The quest for miniaturization has however exposed other problems. For example, reducing battery size means compromising on available power, because the power-to-weight ratio is determined by battery materials and technology.

Transmitting power wirelessly (which would reduce battery size) today requires bulky antennae that do not conform to the human body. Also, implanted or ingested batteries need to be isolated from body tissues, as they can contain toxic metals. And for implanted devices, battery replacement may need surgical intervention (as is the case for cardiac pacemakers). Moreover, even when arrays of active electronic components such as transistors can be made mechanically compliant with soft tissue, the electronics need to be isolated from bodily fluids to avoid current leakage into the tissue as well as performance instabilities resulting from device degradation.

Three Articles in this issue report significant steps towards overcoming these limitations. Nadeau et al. (article no. 0022) demonstrate that the power source for an ingestible bioelectronic device need not be a conventional battery or a wireless

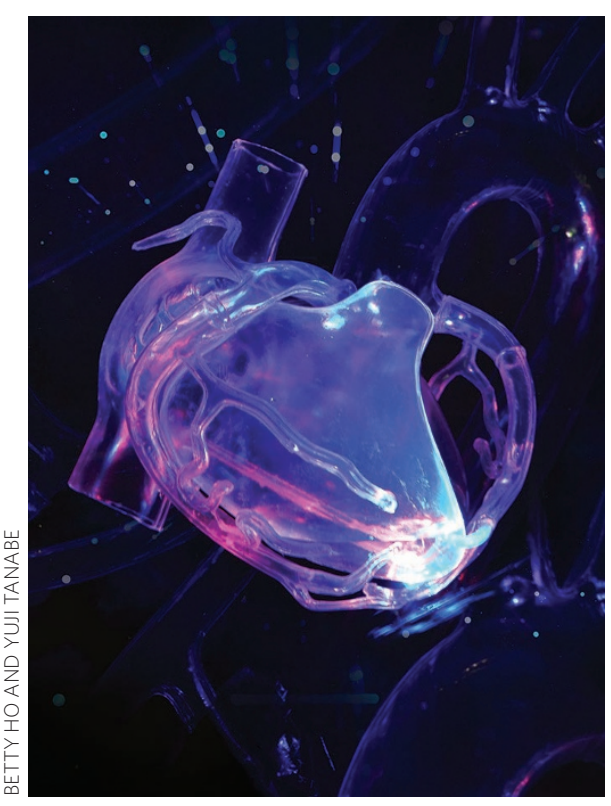

antenna. In a proof-of-concept device for the continuous sensing and wireless transmission of body temperature in the gastrointestinal tract, the researchers show that power can be harvested from the gastric fluids in a pig's stomach for over six days by using an optimized biocompatible galvanic cell. Agrawal et al. (article no. 0043) optimized the wireless transmission of power through tissue to a cardiac-stimulator microdevice implanted into a pig's heart by designing surfaces, loaded with resonant and reactive elements, that focus an electromagnetic field to the microdevice and that conform to the pig's chest and accommodate natural motion. And Fang et al. (article no. 0038) show that a layer of silicon dioxide, less than $1 \mu \mathrm{m}$ thick, thermally grown on a silicon wafer dramatically improves the durability of flexible arrays of transistors fabricated from the passivated wafer when they are put in contact with tissue, as demonstrated by the continuous mapping of the electrical activity of beating rabbit hearts ex vivo and of the rat auditory cortex in vivo (leakage current from the arrays remained extremely low for weeks). The passivating silicon dioxide layer coating the electronics also acts as a signal transducer by capacitively coupling the tissue and semiconductor electronics.
These advances highlight the need for the stable and long-term operation of bioelectronic devices that need to be implanted or ingested. In a Comment (article no. 0049), Dae-Hyeong Kim and co-authors note that implantable electronic devices for diagnostic or therapeutic procedures need to become smaller, more energy-efficient and operate safely through their lifetime. Yet, as discussed in three News \& Views (article nos 0048, 0050 and 0051), it will be challenging to scale down devices while maintaining energy-transfer efficiency and avoiding the foreign-body response and device degradation, and to produce enough power for transmitting high-definition endoscopic images in real time or for operating a miniaturized biopsy module, for example. Wireless transfer of data, and either the harvesting or the wireless transmittance of power continuously, would thus seem imperative needs for future small devices that require minimally invasive surgical intervention and the relay of information to device users or their health carers.

Devices that need to be in contact with tissue, such as transistor arrays for cardiac electrophysiology and conformal surfaces for electromagnetic-field focusing, also need to be flexible, stretchable, and match the compliance of the contacting tissue. These properties are at odds with those of the typically stiff and hard electronics components. Yet softness and flexibility can be achieved by using flexible substrates, strain-compatible fabrication techniques, and by designing open architectures that tolerate deformation (J. Kim et al., Adv. Funct. Mater. 25, 4761-4767; 2015).

Ultimately, bioelectronic devices will be incorporated in closed-loop systems that are able to both monitor and treat the patient. Nadeau et al. also show in preliminary in vitro experiments with physiologic gastric fluid that the energy harvested by the ingestible galvanic cell could in principle be used for targeted therapy, by triggering the electrochemical corrosion of a gold membrane that seals a reservoir containing a model drug. The realization of such a self-powered ingestible device with sensing and therapeutic functions in vivo would make Feynman's vision easy to swallow. 